\title{
A Study on Competitiveness in the Aircraft Industry
}

\author{
Jae-Sung Lee \\ Professor, Department of Trade, Dongeui University, Busan, Korea \\ E-mail: jslee7@deu.ac.kr
}

Received: June 12, 2019. Revised: September 10, 2019. Accepted: September 17, 2019.

\begin{abstract}
Purpose - This study shows that which country has more competitiveness in aerospace industry. The reason why author choose aerospace industry for research is aerospace industry is one of major business in every country in the world instead of infant industry.

Research design and methodology - In order to research this topic, there are 3 tools to analyze competitiveness in aerospace industry. To achieve analysis of competitiveness between USA and England, UN comtrade program which provides huge database including time serial data.

Results - All of TSI are over zero (0) which means that from 2000 to 2018, USA aerospace industry is export specialization as USA aerospace industry has comparative advantage against England aerospace industry. All of TSI in USA are approaching to figure +1 as export specialization except 2010.

Conclusions - Even though RCA value in 2000 is 6.313, however, when time goes by and they are 8.997 in 2005, 8.007 in 2010 and 8.389 in 2015 respectively and RCA value is slightly going down as figure 7 in 2018 . we review above market share analysis data, USA has overpoweringly superior competitive power against British aerospace industry.
\end{abstract}

Keywords: Revealed Comparative Advantage, Trade Specialization Index, Market Share

JEL Classification Code: F17. 


\section{Introduction}

England aerospace industry is evaluated one of the most successful industries among England manufacturing industries and it is marking $0.8 \%$ of total England value added amount in 2018 , of which is scaled $4 \%$ based on only England manufacturing industries. Even though overall England manufacturing industries was recorded trade deficit at the same year, aerospace industry was recorded trade surplus of 2.5 billion British pound. England aerospace industry is dominated approximately 5\% of total production of England manufacturing industry and it is approximately 5.5billion British pound of overall British industry's total value added amount as a one of major business industries, of which showed by Arminen, Koskela and Palukka (2014).

Usually general developed countries have their own major industries such as ship building industry, automobile industry, IT industry, electronic industry and aerospace industry. Each country has been investing a lot of national finance into this kind of major industries that is expressed by Bazeley (2006).

These major industries in each developed country could create tremendous high value-added amount for their countries, of which improve not only national dignity but also increase national wealth. The major industries in each country are not accomplished just one day. When we look into industries in developing countries, we can easily find out those are infant industries in almost every industry in developing countries.

Those infant industries are improved and strengthened after time passed by their own survival strategy including government's subsidies and supports and these developing countries are gradually grown up to developed countries. In this point of time, we need to know how infant industry in developing countries could convert into major industry.

Furthermore, to analyze and to compare these strong major industries between 2 countries is meaningful itself as both 2 countries have already been investing huge financial volume into these major industries. That is the reason why this research should be conducted to analyze which country has genuine competitiveness. The previous research is conducted about aero industry by Koskela, Arminen and Palukka (2013). However, this research is simple comparative analysis instead of competitiveness in aero industry. That is this research is more comparative advantage against those of general research papers and this point is what this research should be conducted with concentration.

\section{Trend of British aerospace industry}

As employment creating effect is high, currently, approximately 255,000 persons are working for aerospace industry. Due to huge plant establishment investment are achieved in 2016, it is expected that minimum over $10 \%$ working employee of increase degree in 2020. It is pointed out as a most big weak point that productivity rate of British aerospace industry is lower than those of other competitive countries.

Even though British aerospace industry is generally, equipped with high productivity comparing to other British manufacturing industries, it is common point of view in this business field that British aerospace is lower level compared to those of major competitive countries such as USA. Additionally, due to the world the highest-level labor, establishment and materials cost, the points that many of enterprises look into foreign countries as well as newly industrialized country's pursuit are the target that British aerospace industry has to solve.

In order to overcome these kinds of weak points, the scale for British aerospace industry's R\&D investment amount is top 2nd position, next rank to pharmaceutical industry in England as they push into fully continued investments to maintain superiority of technology power. The R\&D investment amounts for British aerospace enterprises are larger scale than those of most foreign competitive enterprises.

Top R\&D investment rank for the world major aerospace enterprises in 2018 holds 4 British enterprises (RollsRoyce, Cobham, Smiths, BAE Systems) within top 20 ranks, especially, BAE Systems boast the world 2nd R\&D investment scale which is next to Italian enterprise, Finmeccanica as top 1 position. Even though British government support to aerospace industry's R\&D has been decreased for couple of years due to the matter of budget, brand new support policies in various fields including the readjustment for DTI budget support program are realized suggested by Balassa (1965).

The research \& development costs that aerospace enterprises have invested are tax-exemption together with "Repayable Launch Investment(RLI) policy support. 1/4 among 60million pounds that DTI Technology Programme Funding allocated was used up for aerospace industry's support which implies that the budget support will be increased in the near future. 


\section{Status for aerospace industry}

\subsection{The scale of British aerospace industry}

The scale of British aerospace industry has been holp-up in 2001 due to 911 aircraft terror attack including influence of economic recession. It is generally overall continuous upward trend since 2002 and the $5 \%$ growth rate for aviation industry's sales scale was recorded in 2004, moreover, in 2005, the sales figure was 22.7billion pounds which was as many as $25 \%$ increased.

This figure indicates that the British aerospace industry returns to normal position from the period of the British aerospace industry's hold-up is also insisted by Finlay, Walton and Antaki (2011). The British aviation industry has been dominating approximately $10 \%$ among the world business supply. According to the associated experts in the British aerospace industry, it is expected that the world aerospace industry will be $110 \%$ growth rate based on current sales scale until 2025.

The British aerospace industry is industry that trade surplus has been continued for past 20 years. Among aerospace industry, it is estimated that 130 180 seat aircraft will be dominated the largest rate and this kinds of aircraft will be over $93 \%$ increased based on current figure. Considering growth rate on each seat sized aircraft, it is expected that 70 90 seat aircraft will be 317\% increase rate until 2025 that will show the fastest growth rate. In terms of large scale 300 350 seat aircraft, it is estimated that approximately $247 \%$ increase rate which is caused by Asia-Pacific local area's a lot of demand

Table 1: Prospect for aircraft development (per each size) 2005-2025

\begin{tabular}{|c|c|c|c|c|c|}
\hline Size & 2005(unit) & share $(\%)$ & 2025(unit) & share $(\%)$ & variating rate $(\%)$ \\
\hline 30 50seat aircraft & 3,489 & 11 & 2,175 & 3 & -38 \\
\hline 70 90seat aircraft & 1,164 & 4 & 4,858 & 7 & 317 \\
\hline 110seat jet plane & 1,897 & 6 & 2,953 & 4 & 56 \\
\hline $130 \sim 180$ seat jet plane & 8,353 & 25 & 16,111 & 23 & 93 \\
\hline 200 250seat jet plane & 1,382 & 4 & 2,342 & 3 & 69 \\
\hline 300 350seat jet plane & 1,082 & 3 & 3,751 & 5 & 247 \\
\hline over 400 seat jet plane & 641 & 2 & 1,189 & 2 & 85 \\
\hline Ultra-light jet plane & 0 & 0 & 7,499 & 11 & $\mathrm{n} / \mathrm{a}$ \\
\hline small sized business jet plane & 6,329 & 19 & 8,169 & 12 & 29 \\
\hline medium sized business jet plane & 5,397 & 16 & 11,102 & 16 & 106 \\
\hline large sized business jet plane & 1,494 & 5 & 5,569 & 8 & 273 \\
\hline air cargo aircraft & 1,634 & 5 & 3,403 & 5 & 108 \\
\hline overall aircraft & 32,862 & 100 & 69,119 & 100 & 110 \\
\hline
\end{tabular}

Source: Rolls-Royce data

According to market survey authority, IBIS World, total sales amount of the British aviation industry reached 31.3 billion pounds in 2017-2018 and it is estimated that annual growth rate is 5.3\% during 2013-2018.

Based on demand from civilian aircraft and governmental military purposed aircraft, it is expected that 36 billion pound sales amount will be recorded during 2022 2023 and 2.8\% annual growth rate will be estimated during 20182023. 
The export of aviation industry will be expected $4.7 \%$ increase annually from 2018 to 2023 due to defense budget increase caused by world political tension and GBP value depreciation.

The import of aviation industry will be expected annually $2.3 \%$ increase according to import increase caused by aerospace industry's growth showed by Hindmarsh and Llewellyn (2010).

\subsection{Import trend from top 10 countries}

Table 2: Propeller \& Other components

(Unit: US\$ 1000, \%)

\begin{tabular}{|c|c|c|c|c|}
\hline Rank & Country & $2015($ share $)$ & $2016($ share $)$ & $2017($ share $)$ \\
\hline 1 & USA & $93,374(49.24)$ & $92,369(58.80)$ & $132,073(65.90)$ \\
\hline 2 & Japan & $27,801(14.06)$ & $13,732(8.74)$ & $15,915(7.94)$ \\
\hline 3 & Italia & $17,128(9.03)$ & $12,340(7.86)$ & $11,796(5.89)$ \\
\hline 4 & Canada & $10,663(5.62)$ & $5,892(3.75)$ & $5,680(2.83)$ \\
\hline 5 & France & $3,307(1.74)$ & $4,896(3.12)$ & $5,509(2.75)$ \\
\hline 6 & Belgium & $207(0.11)$ & $818(0.52)$ & $4,705(2.35)$ \\
\hline 7 & Netherland & $12,741(6.72)$ & $6,430(4.09)$ & $3,532(2.26)$ \\
\hline 8 & Ethiopia & $1,020(0.54)$ & $1,680(1.07)$ & $2,658(1.83)$ \\
\hline 9 & Saudi-arabia & $752(0.40)$ & $114(0.07)$ & $2,985(1.49)$ \\
\hline 10 & Germany & $2,522(1.33)$ & $1,505(0.96)$ & $200,413(100 \%)$ \\
\hline
\end{tabular}

Source: Global Trade Atlas (GTA), Rank is based on 2017

Table 3: Airframe supports \& other components

\begin{tabular}{|c|c|c|c|c|}
\hline Rank & Country & 2015 (Share) & 2016 (share) & 2017 (share) \\
\hline 1 & USA & $376,381(43.38)$ & $361,520(41.91)$ & $380,726(46.82)$ \\
\hline 2 & Canada & $127,210(14.66)$ & $114,055(13.22)$ & $117,681(14.47)$ \\
\hline 3 & France & $87,861(10.13)$ & $103,776(12.03)$ & $63,543(7.81)$ \\
\hline 4 & Japan & $19,598(2.26)$ & $80,304(9.31)$ & $46,923(5.77)$ \\
\hline
\end{tabular}




\begin{tabular}{|c|c|c|c|c|}
\hline 5 & China & $57,547(6.63)$ & $51,023(5.92)$ & $39,836(4.90)$ \\
\hline 6 & Mexico & $37,227(4.29)$ & $36,407(4.22)$ & $38,805(4.77)$ \\
\hline 7 & Arabamerit & $41,500(4.78)$ & $2,634(0.31)$ & $17,763(2.18)$ \\
\hline 8 & India & $1,472(0.17)$ & $9,288(1.08)$ & $14,562(1.79)$ \\
\hline 9 & Germany & $11,135(1.28)$ & $14,892(1.73)$ & $7,965(0.98)$ \\
\hline 10 & Saudi-arabia & $7,221(0.83)$ & $6,044(0.70)$ & $813,128(100 \%)$ \\
\hline
\end{tabular}

Source: Global Trade Atlas (GTA), Rank is based on 2017

Table 4: Components for aircraft or helicopter

\begin{tabular}{|c|c|c|c|c|}
\hline Rank & Country & $2015($ share $)$ & $2016($ share $)$ & (Unit: US\$ 1000, \%) \\
\hline 1 & USA & $1,625,264(39.98)$ & $1,341,208(35.48)$ & $1,372,044(35.76)$ \\
\hline 2 & France & $286,546(7.05)$ & $260,817(6.90)$ & $346,000(9.02)$ \\
\hline 3 & South Korea & $193,429(4.76)$ & $269,551(7.13)$ & $275,790(7.19)$ \\
\hline 4 & Belgium & $227,358(5.59)$ & $254,423(6.73)$ & $275,367(7.18)$ \\
\hline 5 & Germany & $402,849(9.91)$ & $280,094(7.41)$ & $267,385(6.97)$ \\
\hline 6 & Malaysia & $179,655(4.42)$ & $204,686(5.42)$ & $196,366(5.12)$ \\
\hline 7 & Italia & $290,555(7.15)$ & $302,333(8.00)$ & $175,627(4.58)$ \\
\hline 8 & India & $73,228(1.80)$ & $98,570(2.61)$ & $109,731(2.86)$ \\
\hline 9 & Netherland & $73,305(1.80)$ & $76,534(2.02)$ & $91,417(2.38)$ \\
\hline 10 & Polland & $40,343(0.99)$ & $40,790(1.08)$ & $68,986(1.80)$ \\
\hline & Total & $4,065,645(100)$ & $3,779,657(100)$ & $3,837,267(100)$ \\
\hline
\end{tabular}

Source : Global Trade Atlas (GTA), Rank is based on 2017 
Table 5: Import scale \& Import trend from South Korea

\begin{tabular}{|c|c|c|c|c|}
\hline Rank & HS Code \& commodity name & $\begin{array}{c}2015 \\
(\text { share })\end{array}$ & $\begin{array}{c}2016 \\
(\text { share })\end{array}$ & $\begin{array}{c}2017 \\
(\text { share })\end{array}$ \\
\hline- & $\begin{array}{c}8803.10 \\
\text { (propeller \& other components) }\end{array}$ & $34(0.02)$ & $3(0)$ & - \\
\hline 17 & $\begin{array}{c}8803.20 \\
\text { (airframe support \& other components) }\end{array}$ & $\begin{array}{l}2,299 \\
(0.27)\end{array}$ & $\begin{array}{c}4,788 \\
(0.56)\end{array}$ & $(0.49)$ \\
\hline 3 & 8803.30 & $193,429(4.76)$ & $269,551(7.13)$ & $275,790(7.19)$ \\
\hline
\end{tabular}

Source: Global Trade Atlas (GTA), Rank is based on 2017

\subsection{Major competitive enterprise}

Refering to the British aviation industry in 2017-2018, top 2 enterprises have dominated 39.1\% of total sales amount such as Airbus Operations Ltd, BAE Systems plc and Rolls-Royce plc, each 15.2\%, 13.2\% and 10.7\% respectively. Rest $60.9 \%$ shares enterprises such as Bombardier, Cobham, Meggitt, QinetiQ and Ultra Electronics is also suggested by Hutchins and Klausen (1996).

Table 6: Each enterprise's sales amount trend in major aircraft \& its components

\begin{tabular}{|c|c|c|c|c|c|c|}
\hline \multicolumn{1}{|c|}{ (Unit: million pounds) } \\
\hline Major aircraft component enterprise & $\begin{array}{c}\text { Market share } \\
\text { on } 2017\end{array}$ & 2013 & 2014 & 2015 & 2016 & 2017 년 \\
\hline Airbus Operations Ltd & $15.2 \%$ & 3,800 & 3,932 & 3,937 & 4,590 & 4,750 \\
\hline $\begin{array}{c}\text { BAE Systems plc } \\
\text { (aircraft business) }\end{array}$ & $13.2 \%$ & 3,958 & 3,376 & 3,818 & 3,934 & 4,120 \\
\hline $\begin{array}{c}\text { Rolls-Royce plc } \\
\text { (aircraft business) }\end{array}$ & $10.7 \%$ & 3,094 & 2,857 & 2,965 & 3,141 & 3,357 \\
\hline
\end{tabular}

Source: IBIS World

\subsection{Distribution structure, customs tariff rate and import regulation \& certification}

According to aerospace industry report announced by UK national assembly, it has manufacturing \& distribution structure that aviation components \& materials are crossing borders several times due to unifying supply chain through overall EU market, of which is additionally supported by Kitzinger (2011).

Table 7: Customs Tariff rate

\begin{tabular}{|c|c|c|c|c|}
\hline \multicolumn{2}{|c|}{ HS code } & $\begin{array}{c}\text { Import } \\
\text { tariff rate } \\
\text { from Korea }\end{array}$ & General tariff rate & $\begin{array}{c}\text { Tariff rate for the biggest } \\
\text { import country(USA) }\end{array}$ \\
\hline $\begin{array}{c}8803.10 \\
\text { (Propeller, its components) }\end{array}$ & 0010 & $0 \%$ & $\begin{array}{c}\text { differ from each } \\
\text { countries }\end{array}$ & $0 \%$ \\
\cline { 2 - 5 } & 0020 & $0 \%$ & $\begin{array}{c}\text { differ from each } \\
\text { countries }\end{array}$ & $0 \%$ \\
\hline
\end{tabular}




\begin{tabular}{|c|c|c|c|c|c|}
\hline \multirow{2}{*}{$\begin{array}{c}8803.20 \\
\text { (airframe support \& its components) }\end{array}$} & 0090 & $0 \%$ & $2.7 \%$ & $2.7 \%$ & $0 \% *$ \\
\cline { 2 - 6 } & 0010 & $0 \%$ & $\begin{array}{c}\text { differ from each } \\
\text { countries }\end{array}$ & \multicolumn{2}{|c|}{$0 \%$} \\
\cline { 2 - 6 } & 0020 & $0 \%$ & $\begin{array}{c}\text { differ from each } \\
\text { countries }\end{array}$ & \multicolumn{2}{|c|}{$0 \%$} \\
\hline $\begin{array}{c}8803.30 \\
(\text { Other components out of aircraft \& } \\
\text { helicopter) }\end{array}$ & 0010 & $0 \%$ & $\begin{array}{c}\text { differ from each } \\
\text { countries }\end{array}$ & \multicolumn{2}{|c|}{$0 \%$} \\
\cline { 2 - 6 } & 0020 & $0 \%$ & $\begin{array}{c}\text { differ from each } \\
\text { countries }\end{array}$ & \multicolumn{2}{|c|}{$0 \%$} \\
\hline
\end{tabular}

Source: Gov. UK

In order to enter into British market, the law of safety standard \& environmental protection law should be preserved strictly and quality regulations certified by international aerospace quality group(IAQA) and international civilian aviation organization(ICAO) should be satisfied is showed by Koschmann (2013).

Military aviation components should be preserved by military aviation management authority and European authority standard and civilian aircraft manufacturing enterprise should be received certification given by European aviation safety authority and civilian aviation authority's requirements.

It needs to pay attention to variety intention for aviation components supply channel. As aircraft \& aircraft components enterprise like Airbus has big dependence towards Europe for manufacturing and assembling final finished one, there is big possibility that customs tariff and customs clearance fee will be occurred when international trade to Europe is conducted after Brexit supported by Melander and Sahlström (2009).

It needs pay attention to possibility that the concerned industry's major enterprises will try to decrease high EU trade dependence and try to variety for components supply channel.

\section{Analysis of aircraft industry between USA-England}

\subsection{Trade Specialization Index for USA-England aircraft Industry}

Table 8: Aerospace industry export from USA to England

\begin{tabular}{|c|c|c|c|c|c|}
\hline Period & Trade Flow & Reporter & Partner & Code & Trade Value \\
\hline 2000 & Export & USA & United Kingdom & 88 & $\$ 4,624,913,294$ \\
\hline 2005 & Export & USA & United Kingdom & 88 & $\$ 5,250,179,709$ \\
\hline 2010 & Export & USA & United Kingdom & 88 & $\$ 5,767,163,285$ \\
\hline 2015 & Export & USA & United Kingdom & 88 & $\$ 9,683,842,537$ \\
\hline 2018 & Export & USA & United Kingdom & 88 & $\$ 11,814,477,433$ \\
\hline
\end{tabular}

Source: calculated by author

Table 9: Aerospace industry export from England to USA

\begin{tabular}{|c|c|c|c|c|c|}
\hline Period & Trade Flow & Reporter & Partner & Code & Trade Value \\
\hline 2000 & Export & United Kingdom & USA & 88 & $\$ 2,435,307,311$ \\
\hline 2005 & Export & United Kingdom & USA & 88 & $\$ 2,321,880,827$ \\
\hline 2010 & Export & United Kingdom & USA & 88 & $\$ 2,812,723,819$ \\
\hline
\end{tabular}




\begin{tabular}{|l|l|l|l|l|l|}
\hline 2015 & Export & United Kingdom & USA & 88 & $\$ 2,814,766,066$ \\
\hline 2018 & Export & United Kingdom & USA & 88 & $\$ 2,972,922,658$ \\
\hline
\end{tabular}

Source: calculated by author

Table 10: USA Trade Specialization Index against England

\begin{tabular}{|c|c|c|c|}
\hline year & $\begin{array}{c}\text { (1) (USA export - } \\
\text { UK export) }\end{array}$ & $\begin{array}{c}\text { (USA export } \\
\text { UK export) }\end{array}$ & TSI = (1) / (2) \\
\hline 2000 & $2,189,605,983$ & $7,060,220,605$ & 0.31013279974 \\
\hline 2005 & $2,928,298,882$ & $7,572,060,536$ & 0.3867241774 \\
\hline 2010 & $2,954,439,466$ & $8,579,887,104$ & 0.34434479501 \\
\hline 2015 & $6,869,076,471$ & $12,498,608,603$ & 0.54958729321 \\
\hline 2018 & $8,841,554,775$ & $14,787,400,091$ & 0.59791137865 \\
\hline
\end{tabular}

Source: calculated by author

Table 11: England Trade Specialization Index against USA

\begin{tabular}{|c|c|c|c|}
\hline year & $\begin{array}{c}\text { (1)(UK export } \\
\text { USA export) }\end{array}$ & $\begin{array}{c}\text { (2)(UK export }+ \\
\text { USA export) }\end{array}$ & TSI = (1) / (2) \\
\hline 2000 & $-2,189,605,983$ & $7,060,220,605$ & -0.31013279974 \\
\hline 2005 & $-2,928,298,882$ & $7,572,060,536$ & -0.3867241774 \\
\hline 2010 & $-2,954,439,466$ & $8,579,887,104$ & -0.34434479501 \\
\hline 2015 & $-6,869,076,471$ & $12,498,608,603$ & -0.54958729321 \\
\hline 2018 & $-8,841,554,775$ & $14,787,400,091$ & -0.59791137865 \\
\hline
\end{tabular}

Source: calculated by author

According to above USA Trade Specialization Index against England, all of TSI are over zero (0) which means that from 2000 to 2018, USA aerospace industry is export specialization as USA aerospace industry has comparative advantage against England aerospace industry conducted by Nevile (2007). All of TSI in USA are approaching to figure +1 as export specialization except 2010. Nevertheless, The TSI in 2010 is still over figure 0 which means it is export specialization instead of import specialization showed by Roschelle (1992).

On the contrary, when we see above England Trade Specialization Index against USA, all of TSI is below zero (0) which means that the British aerospace industry from 2000 to 2018 is import specialization as England aerospace industry does not have comparative advantage against USA aerospace industry is also insisted by Stokoe (2011).

\subsection{RCA analysis Index for USA-England Aircraft Industry}

Table 12: Aerospace industry export from USA to England

\begin{tabular}{|c|c|c|c|c|c|}
\hline Period & Trade Flow & Reporter & Partner & Code & Trade Value \\
\hline 2000 & Export & USA & United Kingdom & 88 & $\$ 4,624,913,294$ \\
\hline 2005 & Export & USA & United Kingdom & 88 & $\$ 5,250,179,709$ \\
\hline
\end{tabular}




\begin{tabular}{|l|l|l|l|l|l|}
\hline 2010 & Export & USA & United Kingdom & 88 & $\$ 5,767,163,285$ \\
\hline 2015 & Export & USA & United Kingdom & 88 & $\$ 9,683,842,537$ \\
\hline 2018 & Export & USA & United Kingdom & 88 & $\$ 11,814,477,433$ \\
\hline
\end{tabular}

Table 13: Aerospace industry export to world market

\begin{tabular}{|c|c|c|c|c|r|}
\hline Period & Trade Flow & Reporter & Partner & Code & \multicolumn{1}{|c|}{ Trade Value } \\
\hline 2000 & Export & world & world & 88 & $\$ 110,669,154,649$ \\
\hline 2005 & Export & world & world & 88 & $\$ 153,597,556,514$ \\
\hline 2010 & Export & world & world & 88 & $\$ 224,052,464,078$ \\
\hline 2015 & Export & world & world & 88 & $\$ 331,571,911,935$ \\
\hline 2018 & Export & world & world & 88 & $\$ 243,337,368,803$ \\
\hline
\end{tabular}

Table 14: USA's total export amount to England

\begin{tabular}{|c|c|c|c|c|c|}
\hline Period & Trade Flow & Reporter & Partner & Code & Trade Value \\
\hline 2000 & Export & USA & United Kingdom & TOTAL & $\$ 41,569,587,474$ \\
\hline 2005 & Export & USA & United Kingdom & TOTAL & $\$ 38,560,827,598$ \\
\hline 2010 & Export & USA & United Kingdom & TOTAL & $\$ 48,328,088,965$ \\
\hline 2015 & Export & USA & United Kingdom & TOTAL & $\$ 56,103,379,996$ \\
\hline 2018 & Export & USA & United Kingdom & TOTAL & $\$ 66,209,113,405$ \\
\hline
\end{tabular}

Table 15: World product total export amount

\begin{tabular}{|c|c|c|c|c|c|}
\hline Period & Trade Flow & Reporter & Partner & Code & Trade Value \\
\hline 2000 & Export & world & world & TOTAL & $\$ 6,280,112,853,131$ \\
\hline 2005 & Export & world & world & TOTAL & $\$ 10,150,157,059,117$ \\
\hline 2010 & Export & world & world & TOTAL & $\$ 15,034,212,250,570$ \\
\hline 2015 & Export & world & world & TOTAL & $\$ 16,116,712,367,922$ \\
\hline 2018 & Export & world & world & TOTAL & $\$ 10,773,408,667,815$ \\
\hline
\end{tabular}


Table 16: Revealed Comparative Advantage in USA-UK aerospace industry

\begin{tabular}{|c|c|c|c|}
\hline Year & $\begin{array}{c}\text { 1) (Aerospace industry } \\
\text { export from USA to } \\
\text { England /Aerospace } \\
\text { industry export to } \\
\text { world market) }\end{array}$ & $\begin{array}{c}\text { (2) (USA's total export } \\
\text { amount to England/ } \\
\text { World product total } \\
\text { export amount) }\end{array}$ & RCA = 1) / (2) \\
\hline 2000 & 0.04179044566 & 0.00661924211 & 6.31347893996 \\
\hline 2005 & 0.03418140124 & 0.00379903753 & 8.99738446122 \\
\hline 2010 & 0.02574023593 & 0.00321454082 & 8.00743788035 \\
\hline 2015 & 0.02920585909 & 0.00348106852 & 8.38991215548 \\
\hline 2018 & 0.0485518418 & 0.00614560493 & 7.90025430418 \\
\hline
\end{tabular}

Source: calculated by author

Conclusively, when we review above RCA value, we can have absolutely strong confidence that USA aerospace industry has overwhelmingly comparative advantage against that of the British aerospace industry.

Even though RCA value in 2000 is 6.313, however, when time goes by and they are 8.997 in 2005, 8.007 in 2010 and 8.389 in 2015 respectively and RCA value is slightly going down as figure 7 in 2018 which is showed by Tuccio, et al (2016). Nevertheless, the RCA value in 2018 is still very high and USA aerospace industry has overpoweringly comparative advantage rather than British aerospace industry.

Generally, we understand that a certain industry in one country has comparative advantage when RCA value is over figure 1. If RCA value of a certain industry in one country is under figure 1, we call this industry has comparative disadvantage because the standard criteria is figure 1 in terms of RCA based on Uncomtrade (2017). That's why RCA values are $6,8,7$ throughout research period which means mentioned industry has very strong comparative advantage against counterpart country.

\subsection{Comparative Competitiveness for Market share for Aircraft Industrial Structure between USA and England}

Table 17: USA Aerospace Industry Export Amount to World

\begin{tabular}{|c|c|c|c|c|l|}
\hline Period & $\begin{array}{c}\text { Trade } \\
\text { Flow }\end{array}$ & Reporter & Partner & HS Code & \multicolumn{1}{|l|}{ Trade Value } \\
\hline 2000 & Export & USA & world & 88 & $\$ 41,044,092,019$ \\
\hline 2005 & Export & USA & world & 88 & $\$ 63,500,882,031$ \\
\hline 2010 & Export & USA & world & 88 & $\$ 79,617,922,992$ \\
\hline 2015 & Export & USA & world & 88 & $\$ 131,627,865,014$ \\
\hline 2018 & Export & USA & world & 88 & $\$ 139,098,077,245$ \\
\hline
\end{tabular}

Source: Calculated by author 
Table 18: British Aerospace Industry Export Amount to World

Unit: USD

\begin{tabular}{|c|c|c|c|c|l|}
\hline Period & $\begin{array}{c}\text { Trade } \\
\text { Flow }\end{array}$ & Reporter & Partner & HS Code & \multicolumn{1}{|c|}{ Trade Value } \\
\hline 2000 & Export & UK & world & 88 & $\$ 9,981,034,697$ \\
\hline 2005 & Export & UK & world & 88 & $\$ 10,937,867,504$ \\
\hline 2010 & Export & UK & world & 88 & $\$ 13,804,991,218$ \\
\hline 2015 & Export & UK & world & 88 & $\$ 18,999,600,292$ \\
\hline 2018 & Export & UK & world & 88 & $\$ 19,018,871,564$ \\
\hline
\end{tabular}

Source: Calculated by author

Table 19: World Total Aerospace Industry Export Amount

Unit: USD

\begin{tabular}{|c|c|c|c|c|c|}
\hline Period & Trade Flow & Reporter & Partner & Code & Trade Value \\
\hline 2000 & Export & world & world & 88 & $\$ 110,669,154,649$ \\
\hline 2005 & Export & world & world & 88 & $\$ 153,597,556,514$ \\
\hline 2010 & Export & world & world & 88 & $\$ 224,052,464,078$ \\
\hline 2015 & Export & world & world & 88 & $\$ 331,571,911,935$ \\
\hline 2018 & Export & world & world & 88 & $\$ 243,337,368,803$ \\
\hline
\end{tabular}

Source: Calculated by author

Table 20: Market Share for 2 country's Aerospace Industry

Unit: (\%)

\begin{tabular}{|c|c|c|c|c|}
\hline & & USA & England & \\
\hline Period & Trade Flow & $\begin{array}{c}\text { (US aerospace export } \\
\text { amount to world/world } \\
\text { total aerospace export } \\
\text { amount) }\end{array}$ & $\begin{array}{c}\text { (UK aerospace export } \\
\text { amount to world/world } \\
\text { total aerospace export } \\
\text { amount) }\end{array}$ & $\begin{array}{c}\text { Competitiveness based on } \\
\text { market share }\end{array}$ \\
\hline 2000 & Export & 0.37087201171 & 0.09018804498 & USA \\
\hline 2005 & Export & 0.41342377751 & 0.07121120773 & USA \\
\hline 2010 & Export & 0.35535392712 & 0.06161499395 & USA \\
\hline 2015 & Export & 0.39698134937 & 0.05730159766 & USA \\
\hline 2018 & Export & 0.57162645396 & 0.07815844996 & USA \\
\hline
\end{tabular}

Source: Calculated by author 
When we review above market share analysis data, USA has overpoweringly superior competitive power against British aerospace industry. Per USA market shares, 0.370 in 2000, 0.413 in 2005, 0.355 in 2010, 0.396 in 2015 and 0.571 in 2018, on the other hand, in terms of the British market shares, 0.090 in 2000, 0.071 in 2005, 0.061 in 2010, 0.057 in 2015 and 0.078 in 2018 surveyed by Tuccio and Nevile (2017). We can easily find out that USA has competitive power over 1-digit figure against England.

\section{Conclusion}

All of TSI are over zero (0) which means that from 2000 to 2018, USA aerospace industry is export specialization as USA aerospace industry has comparative advantage against England aerospace industry. All of TSI in USA are approaching to figure +1 as export specialization except 2010.

When we review above RCA value, we can have absolutely strong confidence that USA aerospace industry has overwhelmingly comparative advantage against that of the British aerospace industry. Even though RCA value in 2000 is 6.313, however, when time goes by and they are 8.997 in 2005, 8.007 in 2010 and 8.389 in 2015 respectively and RCA value is slightly going down as figure 7 in 2018.

we review above market share analysis data, USA has overpoweringly superior competitive power against British aerospace industry. Per USA market shares, 0.370 in 2000, 0.413 in 2005, 0.355 in 2010, 0.396 in 2015 and 0.571 in 2018, on the other hand, in terms of the British market shares, 0.090 in 2000, 0.071 in 2005, 0.061 in 2010, 0.057 in 2015 and 0.078 in 2018 .

This study has used 3 kinds of analysis tool such as "trade specialization index", "revealed comparative advantage index" and "market share index" All of research achievements based on above 3 research analysis tools turn out same results as USA aerospace industry has overwhelmingly comparative advantage and USA aerospace industry has export specialization instead of import specialization which means that USA aerospace industry has strong competitiveness against that of British aerospace industry. This research is unique and creative analytical research method with 3 kinds academic mathematical theories which is never used in this field research.

Nevertheless, this research has research limitations because aero industry itself is one of major industries in each country. It is pretty much difficult to get overseas data about their aero industry as one of important business resources, consequently, both 2 countries reluctantly open their data to world aero industry market. That is one of crucial assignment that I have to overcome these kind of barriers in the near future research tasks.

\section{Reference}

Arminen, I., Koskela, I., \& Palukka, H. (2014). Multimodal production of second pair parts in air traffic control training. Journal of Pragmatics, 6(5), 46-62.

Bazeley, P. (2006). The contribution of computer software to integrating qualitative and quantitative data and analyses. Research in the Schools, 13(1), 64-74.

Balassa B. (1965). Trade liberalization and revealed comparative advantage. Manchester School of Economic and Social Studies, 33(1), 99-123.

Finlay, W. M. L., Walton, C., \& Antaki, C. (2011). Giving feedback to care staff about offering choices to people with intellectual disabilities. In C. Antaki, Applied conversation analysis: Intervention and change in institutional talk, 1(3), 161-183

Hindmarsh, J., \& Llewellyn, N. (2010). Finding organisation in detail: Methodological orientations. In N. Llewellyn \& J. Hindmarsh, Organisation, interaction and practice: Studies in ethnomethodology and conversation analysis, 2(3), 24-46

Hutchins, E., \& Klausen, T. (1996). Distributed cognition in an airline cockpit. In Y. Engeström \& D. Middleton, Cognition and communication at work, (3)1, 15-34

Kitzinger, C. (2011). Working with childbirth helplines: The contributions and limitations of conversation analysis. In C. Antaki, Applied conversation analysis: Intervention and change in institutional talk, 2(3), 98-118

Koschmann, T. (2013). Conversation analysis and collaborative learning. In C. E. Hmelo-Silver, C. Chinn, C. Chan, \& A. O'Donnell, International handbook of collaborative learning, 4(3) 149-167 
Koskela, I., Arminen, I., \& Palukka, H. (2013). Centres of coordination as a nexus of aviation. In P. Haddington, L. Mondada, \& M. Nevile, Interaction and mobility: Language and the body in motion, 15(3), 245-276

Margutti, P. (2010). On designedly incomplete utterances: What counts as learning for teachers and students in primary classroom interaction? Research on Language and Social Interaction, 43(4), 315-345.

Melander, H., \& Sahlström, F. (2009). Learning to fly-The progressive development of situation awareness. Scandinavian Journal of Educational Research, 53(2), 151-166.

Nevile, M. (2007). Talking without overlap in the airline cockpit: Precision timing at work. Text \& Talk $-A n$ Interdisciplinary Journal of Language, Discourse \& Communication Studies, 27(2), 225-249.

Nevile, M. (2010). Looking for action: Talk and gaze home position in the airline cockpit. Australian Review of Applied Linguistics, 33(1), 1-21.

Roschelle, J. (1992). Learning by collaborating: Convergent conceptual change. Journal of the Learning Sciences, 2(3), 235-276.

Stokoe, E. (2011). Simulated interaction and communication skills training: The 'Conversation analytic role-play method'. In C. Antaki, Applied conversation analysis: Intervention and change in institutional talk 3(2), 119139.

Stokoe, E. (2014b). The conversation analytic role-play method (CARM): A method for training communication skills as an alternative to simulated role-play. Research on Language and Social Interaction, 47(3), 255-265.

Tuccio, W. A. (2011). Heuristics to improve human factors performance in aviation. Journal of Aviation/Aerospace Education \& Research, 20(3), 39-53.

Tuccio, W. A., \& Nevile, M. (2017). Using Conversation Analysis in Data-Driven Aviation Training with LargeScale Qualitative Datasets. Journal of Aviation/Aerospace Education \& Research, 26(1). 25-37

Tuccio, W. A., Esser, D. A., Driscoll, G., McAndrew, I., \& Smith, M. O. (2016). Interventionist applied conversation analysis: Collaborative transcription and repair based learning (CTRBL) in aviation. Pragmatics and Society, 7(1), 30-56.

Uncomtrade (2017). Annual Statistics Reports. Retrieved November 21, 2016, from http://comtrade.un.org/ 